Original Article

\title{
Respiratory Failure after Open Descending Aortic Aneurysm Repair: Risk Factors and Outcomes
}

Fumihiro Miyashita, MD, Takeshi Kinoshita, MD, PhD, Tomoaki Suzuki, MD, PhD, and Tohru Asai, MD, PhD

\begin{abstract}
Purpose: This study was conducted to identify predictors of respiratory failure after open repair of descending thoracic aortic aneurysm (DTAA), and to identify any relationship between respiratory failure and long-term survival.

Methods: A total of 75 patients undergoing elective open DTAA repair at the Shiga University of Medical Science Hospital were included in the study. Univariate and multivariate logistic regression analyses were performed to assess the odds ratios for incident postoperative respiratory failure after open DTAA repair. Survival over time was estimated by the Kaplan-Meier method.

Results: Respiratory failure, defined as ventilation dependence for longer than $\mathbf{4 8}$ hours, occurred in 11 patients $(\mathbf{1 4 . 7 \%})$. Independent predictors of respiratory failure after DTAA included prolonged operation time and reduced preoperative forced expiratory volume in $1 \mathrm{~second} /$ forced vital capacity $\times 100\left(\mathrm{FEV}_{1} \%\right)$. In-hospital mortality was higher $(p=0.020)$ among patients with respiratory failure $(18.2 \%$ of those who suffered respiratory failure) than among those without $(0 \%)$. The survival rates at 8 years were significantly lower $(p=\mathbf{0 . 0 1 0})$ in the respiratory failure group (at $44.2 \%$ ) than in the group without respiratory failure (at $89.0 \%$ ).

Conclusion: Lower $\mathrm{FEV}_{1} \%$ and longer operation time were risk factors of postoperative respiratory failure after open repair of DTAA, which in turn is associated with significantly reduced long-term survival.
\end{abstract}

Keywords: descending thoracic aortic aneurysm, open repair, respiratory failure, preoperative pulmonary function, aortic aneurysm

Department of Cardiovascular Surgery, Shiga University of Medical Science, Otsu, Shiga, Japan

Received: January 30, 2020; Accepted: May 1, 2020

Corresponding author: Tomoaki Suzuki, MD, PhD. Department of Cardiovascular Surgery, Shiga University of Medical Science, Setatsukinowa, Otsu, Shiga 520-2192, Japan

E-mail:suzukit@belle.shiga-med.ac.jp

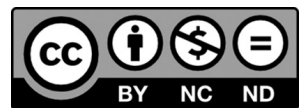

This work is licensed under a Creative Commons Attribution-NonCommercialNoDerivatives International License.

(C)2021 The Editorial Committee of Annals of Thoracic and Cardiovascular Surgery

\section{Introduction}

Although patients requiring thoracic aneurysm repair are increasingly being treated with thoracic endovascular aortic repair (TEVAR), there are many patients who will require open repair for reasons of anatomy, age, or the underlying etiology of their disease. Traditionally, open repair for descending thoracic aortic aneurysms (DTAAs) has been done through left thoracotomy or thoracoabdominal incision. Because such repair involves single-lung ventilation for several hours, these patients 
are particularly prone to developing respiratory complications and even respiratory failure.

Postoperative respiratory failure is among the most serious of the postoperative pulmonary complications. The deleterious influence of pulmonary disease on outcome after open thoracoabdominal aortic aneurysm (TAAA) repair was explored in a previous era. ${ }^{1)}$ However, there is limited information concerning the impact of respiratory function in relation to open DTAA repair. This study was conducted to identify predictors of respiratory failure after open repair of DTAA, and to identify any relationship between respiratory failure and longterm survival.

\section{Materials and Methods}

\section{Patients and study design}

Between January 2002 and May 2019, a total of 132 patients underwent elective and isolated DTAA repair at the Shiga University of Medical Science. Of 132 patients, $51(38.6 \%)$ who underwent endovascular aortic repair were excluded. For the present analysis, 75 patients were included who had preoperative respiratory function evaluated by spirometry ( $92.6 \%$ of elective and isolated cases). All these aortic surgeries were done by two surgeons (T.S. and T.A.). Indications for surgical interventions included descending aortic diameter exceeding $55 \mathrm{~mm}$, rapid dilatation of the aorta $(>5 \mathrm{~mm} /$ 6 months), infection (bronchial/esophageal fistula/stent graft infection). Institutional Review Board approval was obtained (R2019-191) and the need for consent was waived because the study design was retrospective and relevant identifiers were stripped from the data.

\section{Surgical technique}

After induction of general anesthesia, the patient was intubated with a double-lumen endotracheal tube to enable single right-lung ventilation. All operations were done with the patient in the right lateral decubitus position with the pelvis rotated to the left to allow easy access to the left femoral vessels. We do not routinely use cerebrospinal fluid (CSF) drainage, to avoid potential complications of CSF drain insertion. The aorta was accessed through a left thoracoabdominal incision. Resection of adjacent ribs was performed when necessary. Distal aortic perfusion was achieved with femoral-femoral bypass. Hypothermic circulatory arrest (HCA) was used in 27 (36\%) patients and was effected by perfusion cooling. The median cerebral ischemia time during HCA was
41 minutes (interquartile range: $24-50$ ) at a median core temperature of $21.0^{\circ} \mathrm{C}$ (interquartile range: 20.2-22.0). The decision to utilize HCA was prompted by technical considerations, often involving the feasibility and safety of clamping the aorta proximal to the repair. Partial cardiopulmonary bypass without HCA was used in 48 (64\%) patients. Core temperature was targeted at $32^{\circ} \mathrm{C}$ or above (permissive mild hypothermia). In mild hypothermia, we used the segmental clamp technique to reduce spinal cord ischemic time and to close the patient intercostals, which should not be reconstructed immediately after opening the aorta. When internal costal artery (ICA) reconstruction was necessary, the ICAs were occluded with an occlusion catheter to prevent spinal cord steal. The dissection flap extending beyond the distal anastomosis was fenestrated to allow blood flow in both the true and false lumen.

\section{Postoperative respiratory management}

Postoperatively, the double-lumen tube is routinely exchanged for a single-lumen endotracheal tube. We aim to extubate the patients within the first 24 hours and maintain a reverse Trendelenburg position. Chest physiotherapy and nebulizer treatments are routine in the postoperative protocol. In general, if a patient could not be safely separated from the ventilator by approximately 7 days after surgery, a tracheostomy was performed by an open technique.

\section{Definitions}

Preoperative hypertension was defined as history of taking antihypertensive medication and/or receiving nutrition or physical therapy based on the diagnosis of hypertension. Postoperative respiratory failure was defined as ventilator dependence for more than 48 hours in accordance with a previous study of patients undergoing thoracoabdominal aortic aneurysm repair. ${ }^{1)}$ This included reintubation and occasional need for tracheostomy.

\section{Statistical analysis}

Categorical variables are described in numbers and percentages. All reported continuous variables were not normally distributed and therefore were described as medians with interquartile range. Categorical variables were analyzed using the $\chi^{2}$ test or Fisher's exact test and continuous variables using the t-test or nonparametric Mann-Whitney U test as appropriate. Multivariate logistic regression modeling was used to determine independent perioperative predictors of respiratory failure after 
descending aortic repair. Variables that reached $p<0.050$ in the univariate analysis or that were considered clinically important were entered into multivariate logistic regression analysis. For reliable analysis, we required at least 10 events of the primary outcome measure per variate. ${ }^{2)}$ Multivariate logistic regression was used after controlling simultaneously for potential confounders. Cox proportional hazard regression was performed for the analysis of late mortality. Variables that reached $p<0.050$ in the univariate analysis or that were considered clinically important were entered into the multivariate model. Survival over time was estimated by the Kaplan-Meier method and compared in a log-rank test. Results were considered statistically significant at a level of $p<0.050$. Data were extracted into a Microsoft Excel spreadsheet (Microsoft Corp, Redmond, WA, USA) and analyzed in SPSS Statistics 25.0 software (SPSS Inc., Chicago, IL, USA) for Windows (Microsoft Corp).

\section{Results}

\section{Early outcomes}

Respiratory failure requiring ventilation for longer than 48 hours occurred in 11 of 75 patients (14.7\%). Preoperative characteristics are reviewed in Table 1. Patients with such respiratory failure were less likely to have shown hypertension $(36.3 \%$ vs $82.8 \% ; p=0.001)$ than were the patients without respiratory failure. Although there was no statistically significant difference, there were trends toward lower forced expiratory volume in 1 second/forced vital capacity $\times 100\left(\mathrm{FEV}_{1} \%\right)$ and vital capacity in the respiratory failure group than in the group without respiratory failure.

Operative details are shown in Table 1. Patients with respiratory failure had longer operation times (median operation time, 327 minutes [interquartile range: 294 398] vs 252 minutes [interquartile range: 193-296]; $p=0.001$ ), longer bypass times (median bypass time, 173 minutes [interquartile range: $114-198]$ vs $79 \mathrm{~min}-$ utes [interquartile range: 51-138]; $p=0.004)$, were more likely to have undergone circulatory arrest (63.6\% vs $31.3 \% ; p=0.039)$, and required more transfusions (100\% vs $59.4 \%$; $p=0.009)$.

Early outcomes are shown in Table 2. There was zero 30-day mortality in both groups. In-hospital mortality was higher in patients with respiratory failure $(\mathrm{n}=2$, $18.2 \%)$ than in those without $(\mathrm{n}=0,0 \% ; p=0.020)$. One patient, an 82-year-old man, had previously undergone a total arch replacement. He was found to have a distal anastomotic pseudoaneurysm involving the composite graft and a separate descending aortic aneurysm. After the open descending aortic aneurysm repair, he was also found to have an aorto-esophageal fistula. Mediastinitis developed and he subsequently died. A second patient, a 56-year-old man, had previously undergone replacement of his ascending aorta for acute type A dissection. Nine years later, he underwent open descending aortic repair for chronic post-dissection aneurysm. A significant amount of thrombus was found inside the aneurysm. Visceral arterial embolism developed postoperatively, and subsequently he died. Most respiratory complications were significantly more common in patients with respiratory failure than in those without. The length of stay was longer in patients with respiratory failure (median length of stay, 50 days [interquartile range: 31-137] than in those without (median length of stay, 22 days [interquartile range: $17-34] ; p=0.001$ ).

Univariate and multivariate logistic regression analyses of the incidence of postoperative respiratory failure in relation to baseline and operative values is shown in Table 3. In univariate models, hypertension was associated with a decreased risk of postoperative respiratory failure (odds ratio: 0.12; CI: 0.03-0.48; $p=0.003$ ). On the other hand, longer operation time (odds ratio: 1.15 ; CI: $1.05-1.25 ; p=0.003$ ) and use of HCA (odds ratio: 3.85 ; CI: $1.01-14.7 ; p=0.048$ ) were associated with increased risks of postoperative respiratory failure. In multivariate models, hypertension was also associated with a decreased risk of postoperative respiratory failure (odds ratio: 0.06; CI: 0.010.57; $p=0.014$ ). Lower $\mathrm{FEV}_{1} \%$ (odds ratio: 3.69; CI: $1.24-11.0 ; p=0.019$ ) and longer operation time (odds ratio: 1.18 ; CI: $1.03-1.35 ; p=0.017$ ) were statistically significant risk factors of postoperative respiratory failure. These variables jointly yielded an area under the curve (AUC) of 0.937 .

\section{Long-term outcomes}

The median follow-up durations were 1.7 years (interquartile range: $0.5-5.5]$ in the respiratory failure group and 3.0 years (interquartile range: 1.0-7.5] in the group without respiratory failure. In multivariate analysis, included variables were age (per year); hypertension; smoking history; vital capacity (10\% decrement); $\mathrm{FEV}_{1} \%$ (10\% decrement); operation time (10-minute increments); HCA; postoperative respiratory failure. As a result, age (hazard ratio: 1.08; CI: $1.01-1.16 ; p=0.032$ ) and postoperative respiratory failure (hazard ratio: 
Table 1 Preoperative and intraoperative patient demographics

\begin{tabular}{|c|c|c|c|c|}
\hline & $\begin{array}{c}\text { All } \\
(n=75)\end{array}$ & $\begin{array}{l}\text { Respiratory failure } \\
\qquad(\mathrm{n}=11)\end{array}$ & $\begin{array}{l}\text { No respiratory } \\
\text { failure } \\
(\mathrm{n}=64)\end{array}$ & $p$ value \\
\hline \multicolumn{5}{|l|}{ Preoperative } \\
\hline Age, years & $69[57-75]$ & $62[53-80]$ & $69[58-75]$ & 0.595 \\
\hline Age $\geq 70$ years & $32(42.6)$ & $3(27.2)$ & $29(45.3)$ & 0.263 \\
\hline Female gender & $14(18.6)$ & $2(18.1)$ & $12(18.7)$ & 0.964 \\
\hline Body mass index, $\mathrm{kg} / \mathrm{m}^{2}$ & $23.3[20.5-26.0]$ & $22.5[20.5-26.0]$ & 23.4 [20.5-25.9] & 0.784 \\
\hline Smoking history & $54(72.0)$ & $9(81.8)$ & $45(70.3)$ & 0.432 \\
\hline Diabetes mellitus & $12(16.0)$ & $3(27.2)$ & $9(14.0)$ & 0.269 \\
\hline Hypertension & $57(76.0)$ & $4(36.3)$ & $53(82.8)$ & 0.001 \\
\hline Serum creatinine, $\mathrm{mg} / \mathrm{dL}$ & $0.93[0.77-1.29]$ & $0.79[0.69-1.02]$ & $0.99[0.78-1.31]$ & 0.123 \\
\hline $\mathrm{FEV}_{1} \%, \%$ & $71.6[64.8-76.4]$ & $70.3[58.9-74.5]$ & 71.6 [65.6-76.7] & 0.154 \\
\hline Vital capacity, \% & $96.2[83.4-112]$ & $85.9[82.1-116]$ & $97.0[84.6-112]$ & 0.150 \\
\hline Marfan syndrome & $4(5.3)$ & $1(9.1)$ & $3(4.6)$ & 0.477 \\
\hline \multicolumn{5}{|l|}{ Cause } \\
\hline Degenerative aneurysm & $29(38.7)$ & $2(18.2)$ & $27(42.1)$ & 0.131 \\
\hline Chronic post-dissection aneurysm & $41(54.7)$ & $7(63.6)$ & $34(53.1)$ & 0.518 \\
\hline Pseudoaneurysm & $3(4.0)$ & $1(9.1)$ & $2(3.1)$ & 0.383 \\
\hline Re-expansion after TEVAR & $2(2.7)$ & $1(9.1)$ & $1(1.6)$ & 0.274 \\
\hline \multicolumn{5}{|l|}{ Proximal extent of aortic replacement } \\
\hline Zone 3 & $27(36.0)$ & $3(27.2)$ & $24(37.5)$ & 0.514 \\
\hline Zone 4 & $48(64.0)$ & $8(72.7)$ & $40(62.5)$ & 0.514 \\
\hline $\mathrm{T} 4$ & $23(30.7)$ & $6(54.5)$ & $17(26.6)$ & 0.063 \\
\hline T5 & $7(9.3)$ & $1(9.1)$ & $6(9.4)$ & 0.976 \\
\hline T6 & $5(6.7)$ & $0(0)$ & $5(7.8)$ & 0.442 \\
\hline $\mathrm{T} 7$ & $5(6.7)$ & $0(0)$ & $5(7.8)$ & 0.442 \\
\hline $\mathrm{T} 8$ & $3(4.0)$ & $0(0)$ & $3(4.7)$ & 0.617 \\
\hline T9 & $2(2.7)$ & $1(9.1)$ & $1(1.6)$ & 0.274 \\
\hline $\mathrm{T} 10$ & $3(4.0)$ & $0(0)$ & $3(4.7)$ & 0.617 \\
\hline EuroSCORE II, \% & 3.39 [2.11-6.42] & 3.76 [2.88-6.42] & 3.18 [2.08-6.35] & 0.574 \\
\hline Previous aortic repair & $38(50.6)$ & $7(63.6)$ & $31(48.4)$ & 0.352 \\
\hline Open surgery & 45 & 7 & 8 & \\
\hline Aortic root & $1(1.3)$ & $0(0)$ & $1(1.5)$ & 0.853 \\
\hline Ascending aorta & $13(17.3)$ & $3(27.2)$ & $10(15.6)$ & 0.346 \\
\hline Arch & $19(25.3)$ & $4(36.3)$ & $15(23.4)$ & 0.362 \\
\hline Descending aorta & $3(4.0)$ & $0(0)$ & $3(4.7)$ & 0.617 \\
\hline Abdominal aorta & $9(12.0)$ & $0(0)$ & $9(14.0)$ & 0.184 \\
\hline Endovascular repair & 3 & 1 & 2 & \\
\hline Descending aorta & $3(6.0)$ & $1(9.1)$ & $2(3.1)$ & 0.383 \\
\hline \multicolumn{5}{|l|}{ Intraoperative } \\
\hline Operation time, minutes & $266[201-323]$ & 327 [294-358] & 252 [193-296] & 0.001 \\
\hline Bypass time, minutes & $88[54-146]$ & $173[114-198]$ & $79[51-138]$ & 0.004 \\
\hline Hypothermic circulatory arrest & $27(36.0)$ & $7(63.6)$ & $20(31.3)$ & 0.039 \\
\hline Intercostal arteries reconstruction & $5(6.7)$ & $0(0)$ & $5(7.8)$ & 0.442 \\
\hline Transfusion & $49(65.3)$ & $11(100)$ & $38(59.4)$ & 0.009 \\
\hline
\end{tabular}

Categorical data are presented as n (\%) and continuous data as median [interquartile range]. EuroSCORE: European System for Cardiac Operative Risk Evaluation; $\mathrm{FEV}_{1} \%$ = (forced expiratory volume in 1 second/forced vital capacity) $\times 100$; TEVAR: thoracic endovascular aortic repair

5.79; CI: $1.58-21.2 ; p=0.008)$ emerged as significant independent predictors of long-term survival (Table 4). The actual survival rate at 8 years was $44.2 \%$ in the respiratory failure group and $89.0 \%$ in the group without respiratory failure, which was significantly different $(p=0.010$, Fig. 1).

\section{Discussion}

Whereas respiratory failure after TAAA repair occurs in $20 \%-43 \%$ of patients, ${ }^{1,3-5)}$ such information is scarce in open repair for DTAAs isolated to the chest. In our series, $14.7 \%$ of patients developed respiratory failure; 
Table 2 Early outcomes

\begin{tabular}{lcccc}
\hline & $\begin{array}{c}\text { All } \\
(\mathrm{n}=75)\end{array}$ & $\begin{array}{c}\text { Respiratory } \\
\text { failure } \\
(\mathrm{n}=11)\end{array}$ & $\begin{array}{c}\text { No respiratory } \\
\text { failure } \\
(\mathrm{n}=64)\end{array}$ & $p$ value \\
\hline 30-day mortality & $0(0)$ & $0(0)$ & $0(0)$ & - \\
In-hospital mortality & $2(2.7)$ & $2(18.2)$ & $0(0)$ & 0.020 \\
Tracheostomy & $5(6.7)$ & $5(45.5)$ & $0(0)$ & $<0.001$ \\
Pneumonia & $4(5.3)$ & $3(27.3)$ & $1(1.6)$ & 0.009 \\
Pleural effusion requiring drainage & $22(29.3)$ & $8(72.7)$ & $14(21.9)$ & 0.001 \\
Chylothorax & $6(8.0)$ & $2(18.2)$ & $4(6.3)$ & 0.178 \\
Reintubation & $5(6.7)$ & $4(36.4)$ & $1(1.6)$ & 0.001 \\
Stroke & $5(6.7)$ & $2(18.2)$ & $3(4.7)$ & 0.153 \\
Paraplegia or paraparesis & $3(4.0)$ & $1(9.1)$ & $2(3.1)$ & 0.383 \\
Bleeding necessitating reoperation & $5(6.7)$ & $1(9.1)$ & $4(6.3)$ & 0.558 \\
Wound dehiscence & $10(13.3)$ & $2(18.2)$ & $8(12.5)$ & 0.609 \\
Wound infection & $4(5.3)$ & $1(9.1)$ & $3(4.7)$ & 0.477 \\
Length of stay, days & $23[17-39]$ & $50[31-137]$ & $22[17-34]$ & 0.001 \\
\hline
\end{tabular}

Categorical data are presented as $\mathrm{n}(\%)$ and continuous data as median [interquartile range].

Table 3 Univariate and multivariate models of the incidence of postoperative respiratory failure in relation to baseline and operative value

\begin{tabular}{|c|c|c|c|c|}
\hline \multirow[b]{2}{*}{ Variable } & \multicolumn{2}{|c|}{ Univariate model } & \multicolumn{2}{|c|}{ Multivariate model } \\
\hline & $\begin{array}{l}\text { Odds ratio } \\
(95 \% \mathrm{CI})\end{array}$ & $p$ value & $\begin{array}{l}\text { Odds ratio } \\
(95 \% \mathrm{CI})\end{array}$ & $p$ value \\
\hline Age, years (10-year increments) & $0.82(0.49-1.38)$ & 0.453 & $0.70(0.33-1.48)$ & 0.352 \\
\hline \multicolumn{5}{|l|}{ Sex } \\
\hline Female & 1 [Reference] & & & \\
\hline Male & $1.04(0.20-5.44)$ & 0.964 & & \\
\hline Body mass index, $\mathrm{kg} / \mathrm{m}^{2}$ & $1.07(0.91-1.25)$ & 0.416 & & \\
\hline \multicolumn{5}{|l|}{ Diabetes mellitus } \\
\hline No & 1 [Reference] & & & \\
\hline Yes & $2.29(0.51-10.2)$ & 0.279 & & \\
\hline \multicolumn{5}{|l|}{ Hypertension } \\
\hline No & 1 [Reference] & & 1 [Reference] & \\
\hline Yes & $0.12(0.03-0.48)$ & 0.003 & $0.06(0.01-0.57)$ & 0.014 \\
\hline \multicolumn{5}{|l|}{ Dyslipidemia } \\
\hline No & 1 [Reference] & & & \\
\hline Yes & $0.19(0.02-1.59)$ & 0.126 & & \\
\hline \multicolumn{5}{|l|}{ Previous aortic repair } \\
\hline No & 1 [Reference] & & & \\
\hline Yes & $1.86(0.50-6.99)$ & 0.357 & & \\
\hline \multicolumn{5}{|l|}{ Marfan syndrome } \\
\hline No & 1 [Reference] & & & \\
\hline Yes & $2.03(0.19-21.5)$ & 0.556 & & \\
\hline Preoperative Serum creatinine, $\mathrm{mg} / \mathrm{dL}$ & $0.69(0.20-2.36)$ & 0.552 & & \\
\hline EuroSCORE II, \% & $1.02(0.88-1.18)$ & 0.773 & & \\
\hline \multicolumn{5}{|l|}{ Smoking history } \\
\hline No & 1 [Reference] & & 1 [Reference] & \\
\hline Yes & $1.90(0.38-9.63)$ & 0.438 & $15.7(0.89-278)$ & 0.060 \\
\hline Vital capacity, \% (10\% decrement) & $1.26(0.90-1.77)$ & 0.181 & $1.45(0.92-2.30)$ & 0.113 \\
\hline $\mathrm{FEV}_{1} \%, \%$ (10\% decrement) & $1.55(0.88-2.71)$ & 0.129 & $3.69(1.24-11.0)$ & 0.019 \\
\hline Operation time, minutes (10-minute increments) & $1.15(1.05-1.25)$ & 0.003 & $1.18(1.03-1.35)$ & 0.017 \\
\hline \multicolumn{5}{|l|}{ Hypothermic circulatory arrest } \\
\hline No & 1 [Reference] & & 1 [Reference] & \\
\hline Yes & $3.85(1.01-14.7)$ & 0.048 & $1.46(0.18-11.9)$ & 0.723 \\
\hline
\end{tabular}

EuroSCORE: European System for Cardiac Operative Risk Evaluation; FEV $\%$ : (forced expiratory volume in 1 second/forced vital capacity) $\times 100$ 
Table 4 Multivariate analysis of risk factors for long-term survival

\begin{tabular}{lcc}
\hline Variable & Hazard ratio $(95 \% \mathrm{CI})$ & $p$ value \\
\hline Age, years (per year) & $1.08(1.01-1.16)$ & 0.032 \\
Hypertension & $0.70(0.09-5.80)$ & 0.745 \\
Smoking history & $1.31(0.20-8.57)$ & 0.781 \\
Vital capacity, \% (10\% decrement) & $1.05(0.73-1.51)$ & 0.786 \\
FEV $\%, \%$ (10\% decrement) & $1.04(0.97-1.12)$ & 0.299 \\
Operation time, minutes (10-minute & $1.00(0.99-1.01)$ & 0.656 \\
increments) & & \\
Hypothermic circulatory arrest & $2.67(0.37-19.1)$ & 0.328 \\
Postoperative respiratory failure & $5.79(1.58-21.2)$ & 0.008 \\
\hline
\end{tabular}

$\mathrm{FEV}_{1} \%$ : (forced expiratory volume in 1 second/forced vital capacity) $\times 100$

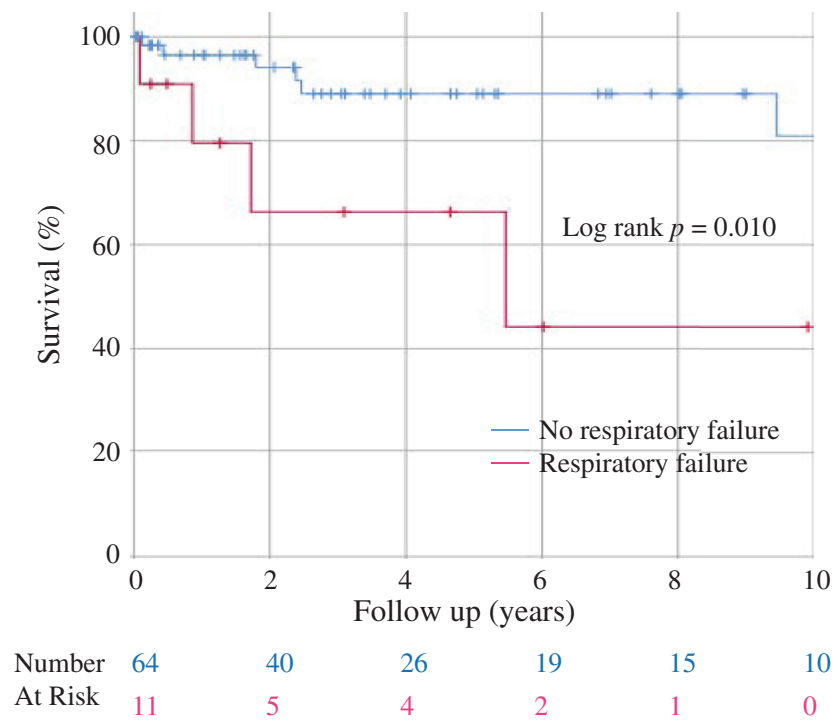

Fig. 1 Kaplan-Meier curves of long-term survival for 75 patients who underwent descending thoracic aortic aneurysm repair, stratified by whether patients developed respiratory failure after repair $(n=11)$ or not $(n=64)$.

this is a lower incidence than in previous findings including open TAAA repair. In addition, univariate and multivariate analyses showed that longer operation time was associated with an increased risk of postoperative respiratory failure, and HCA was also associated with increased risks of postoperative respiratory failure in univariate models. These findings suggest that surgical invasiveness is a risk factor for respiratory failure after open DTAA repair.

Although the risk of respiratory failure was mostly dependent on operative factors, reduced preoperative $\mathrm{FEV}_{1} \%$ was a statistically significant risk factor for postoperative respiratory failure in multivariate models. Girardi and colleagues have reported preoperative $\mathrm{FEV}_{1} \%<50 \%$ as predictive of increased respiratory failure, tracheostomy, and operative mortality in patients undergoing open DTAA or TAAA repair. ${ }^{6}$ With an aging population and an overall increase in the prevalence of chronic obstructive pulmonary disease (COPD), surgeons performing DTAA repair are evaluating increasing numbers of patients with compromised pulmonary function. Upwards of $25 \%$ of a large, contemporary group of patients undergoing open DTAA or TAAA repair had advanced COPD. ${ }^{7}$ In patients with significant pulmonary dysfunction, there may be some distinct advantage of endovascular repair over open repair. The Gore TAG Investigators reported a substantial reduction in postoperative respiratory failure when comparing TEVAR with a historical group of controls undergoing open DTAA repair (4\% vs 20\%). ${ }^{8}$ Similarly, Tanaka and colleagues found that TEVAR had lower risk of postoperative respiratory failure, compared with open DTAA 
repair in an observational study (10\% vs $34 \%) .{ }^{9)}$ However, TEVAR is still associated with significant complications and a relatively high incidence of endoleaks and repeat interventions that may affect its long-term durability. ${ }^{10)}$ We need to select the procedure for DTAA carefully considering these factors.

Interestingly, preoperative hypertension was significantly more common in the group without respiratory failure. Univariate and multivariate analyses also showed that preoperative hypertension was associated with a decreased risk of postoperative respiratory failure. Griepp and colleagues found that a history of hypertension was present in most patients with aneurysms, and it was widely recognized that hypertension, especially diastolic hypertension, was highly correlated with the initial development of aneurysms. ${ }^{11)}$ They also found significantly more rapid growth of aortic aneurysms in smokers and patients with COPD. ${ }^{11)}$ In our series, there were trends toward lower $\mathrm{FEV}_{1} \%$ and more smokers in the respiratory failure group than in the group without respiratory failure. We simply defined all patients receiving antihypertensive treatment as having, or having had, preoperative hypertension, but most such people had regular care, and so were likely to have more-or-less controlled hypertension, and to receive anti-smoking advice and possibly meticulous antiCOPD care where relevant. Therefore, the appearance of association between preoperative hypertension and decreased risk of postoperative respiratory failure may have been an artefact of selection.

Unsurprisingly, our patients with respiratory failure had significantly higher in-hospital mortality and longer hospital stay than patients without respiratory failure. Furthermore, survival estimates at 8 years were significantly lower in the respiratory failure group than in the group without respiratory failure. Several studies have identified early and mid-term consequences of prolonged ventilation after cardiac surgery. ${ }^{12,13)}$ These studies found that prolonged mechanical ventilation was associated with reduced early and mid-term survival; this is consistent with our findings.

This study had certain limitations. It was retrospective, from a single center, and observational. The retrospective nature along with the relatively long time period this study covers may have involved changes in clinical practices and processes that might have affected the outcomes. Also, the sample size was small, with only 75 patients, of whom only 11 suffered respiratory failure.

\section{Conclusion}

Lower $\mathrm{FEV}_{1} \%$ and longer operation time were risk factors for postoperative respiratory failure. Moreover, patients with postoperative respiratory failure had an increased incidence of in-hospital mortality and markedly poorer long-term survival. Endovascular repair (including total arch replacement and subsequent TEVAR, or fenestrated or branched stent placement) may be more suitable therapeutic option in high-risk patients.

\section{Acknowledgment}

We thank Dr. Piers Vigers for reviewing our manuscript.

\section{Disclosure Statement}

The authors have declared that no conflict of interest exists.

\section{References}

1) Svensson LG, Hess KR, Coselli JS, et al. A prospective study of respiratory failure after high-risk surgery on the thoracoabdominal aorta. J Vasc Surg 1991; 14: 271-82.

2) Steyerberg EW, Eijkemans MJ, Harrell FE, et al. Prognostic modeling with logistic regression analysis: in search of a sensible strategy in small data sets. Med Decis Making 2001; 21: 45-56.

3) Money SR, Rice K, Crockett D, et al. Risk of respiratory failure after repair of thoracoabdominal aortic aneurysms. Am J Surg 1994; 168: 152-5.

4) Etz CD, Di Luozzo G, Bello R, et al. Pulmonary complications after descending thoracic and thoracoabdominal aortic aneurysm repair: predictors, prevention, and treatment. Ann Thorac Surg 2007; 83: S870-6; discussion S890-2.

5) Songdechakraiwut T, Aftab M, Chatterjee S, et al. Tracheostomy after thoracoabdominal aortic aneurysm repair: risk factors and outcomes. Ann Thorac Surg 2019; 108: 778-84.

6) Girardi LN, Lau C, Munjal M, et al. Impact of preoperative pulmonary function on outcomes after open repair of descending and thoracoabdominal aortic aneurysms. J Thorac Cardiovasc Surg 2017; 153: S22-9. e2.

7) Kilic A, Shah AS, Black JH III, et al. Trends in repair of intact and ruptured descending thoracic aortic aneurysms in the United States: a population-based analysis. J Thorac Cardiocasc Surg 2014; 147: 1855-60.

8) Bavaria JE, Appoo JJ, Makaroun MS, et al. Endovascular stent grafting versus open surgical repair of 
descending thoracic aortic aneurysms in low-risk patients: a multicenter comparative trial. J Thorac Cardiovasc Surg 2007; 133: 369-77.

9) Tanaka A, Sandhu HK, Pratt WB, et al. Risk modeling to optimize patient selection for management of the descending thoracic aortic aneurysm. Ann Thorac Surg 2018; 105: 724-30.

10) Etezadi V, Schiro B, Peña CS, et al. Endovascular treatment of descending thoracic aortic disease: single-center, 15-year experience. J Vasc Interv Radiol 2012; 23: 468-75.
11) Griepp RB, Ergin MA, Galla JD, et al. Natural history of descending thoracic and thoracoabdominal aneurysms. Ann Thorac Surg 1999; 67: 1927-30; discussion 1953-8.

12) Saleh HZ, Shaw M, Al-Rawi O, et al. Outcomes and predictors of prolonged ventilation in patients undergoing elective coronary surgery. Interact Cardiovasc Thorac Surg 2012; 15: 51-6.

13) Trouillet JL, Combes A, Vaissier E, et al. Prolonged mechanical ventilation after cardiac surgery: outcome and predictors. J Thorac Cardiovasc Surg 2009; 138: 948-53. 